\section{The impact of a dedicated orthoptist to our Acute Stroke Unit (ASU) at St. George's University Hospital - London}

\section{BACKGROUND}

The 2016 stroke national clinical guidelines ${ }^{1}$ emphasised the importance of orthoptic assessment for patients presenting to Acute Stroke Units (ASUs) at St George's, London, UK. The orthoptic society ${ }^{2}$ recommends orthoptic review within the acute admission. A local audit in $2014^{3}$ showed $48 \%$ of patients with a visual problem saw an orthoptist.

\section{METHODS}

Stroke orthoptist (part time - 2 sessions in a week) appointed January 2017. Role of orthoptist: assessed all referrals from the multidisciplinary team on the ASU for patients with a visual deficit. 18/09/17 - 21/12/17 (3 months) data collected on:

- Number of patients who saw orthoptist.

- All patients on ASU issued simplified VFQ-25 questionnaire to examine the difference an orthoptic assessment made to patients' visual quality of life.

\section{RESULTS}

Review of notes

3 months 61 patients admitted to ASU
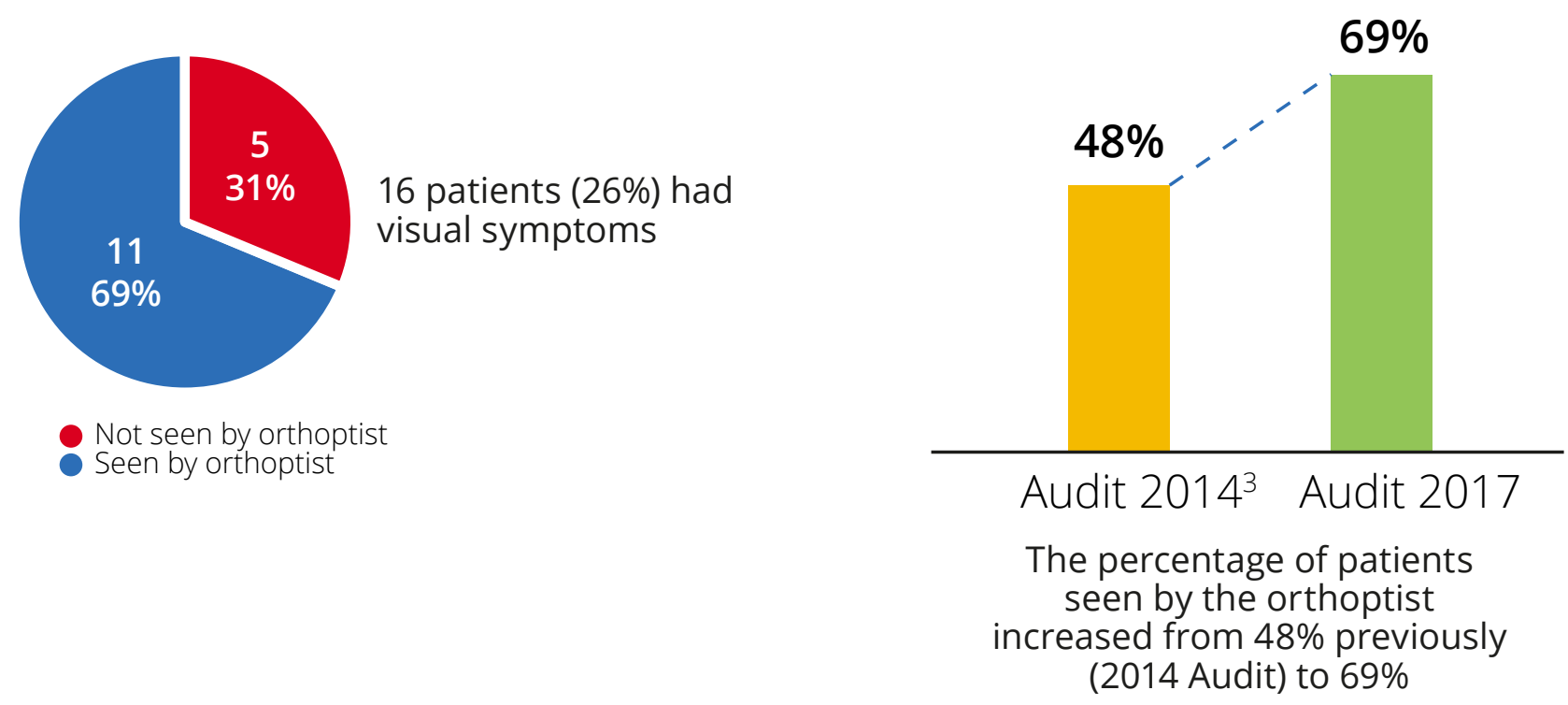

\section{Questionnaire}

24 questionnaires were returned (39\% response rate)

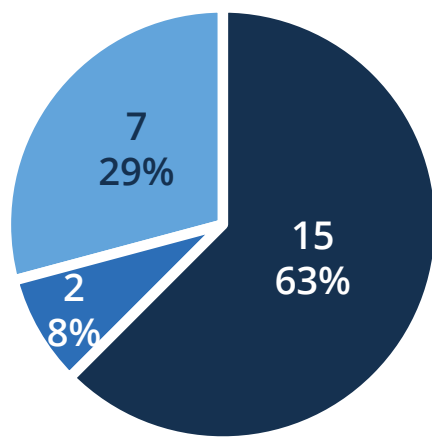

$29 \%$ of patients

reported poor vision

$\approx 26 \%$ of patients

who had visual

symptoms on review

of notes

\section{Review of notes}

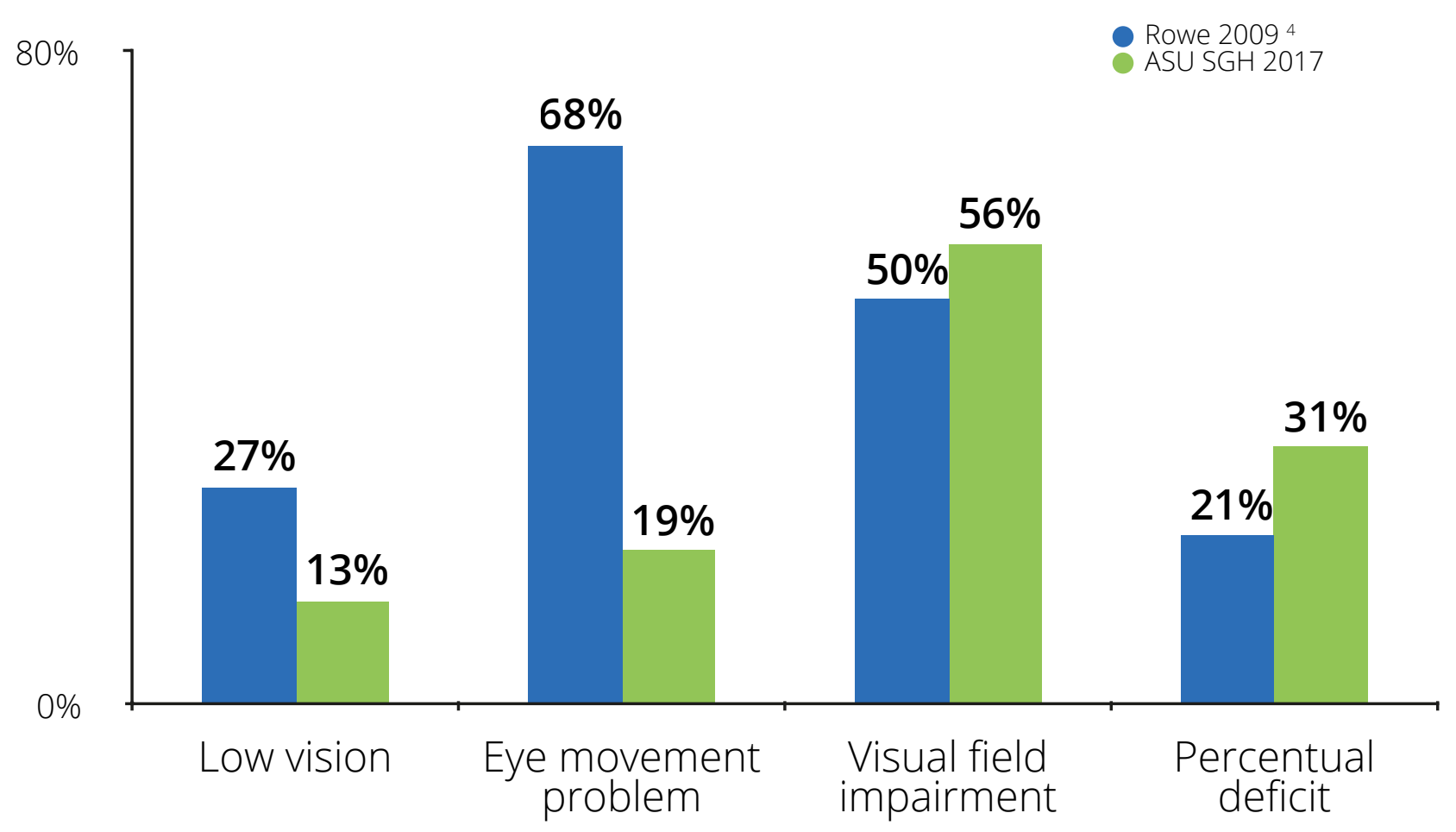

Questionnaire (impact of seeing orthopist)

9 patients who returned questionnaires reported visual concern

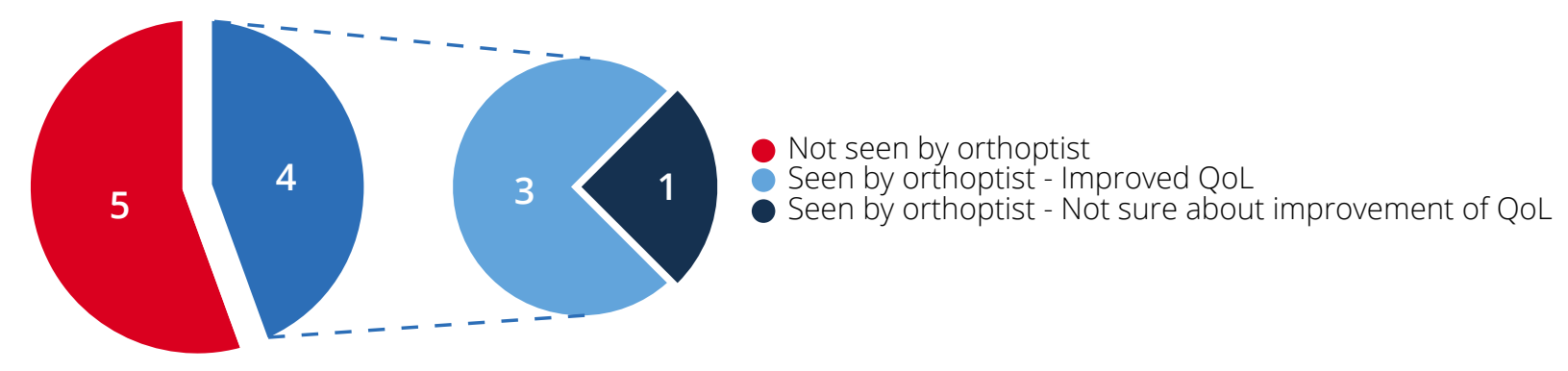

\title{
CONCLUSIONS
}

Appointing a stroke orthoptist has had a significant positive impact on the visual symptoms of stroke patients - almost 70\% are now seen and 75\% of those seen feel that their quality of life has been impacted on positively by this encounter. 4 out of 9 patients reporting visual concerns via questionnaire were seen by the orthoptist (note that 29\% of patients reported poor vision $\approx 26 \%$ of patients who had visual symptoms on review of notes):

- Did some patient see the orthoptist but wrongly answered the questionnaire?

- Under-detection and under-acknowledgement:

- Did the stroke team reckon the orthoptist assessment was not required?

- Did some patient fail to report their visual concerns to the stroke team?

- Did the stroke team fail to refer the patient to the orthoptist?

Neglect is the symptom not seen by orthoptist. Little evidence that orthoptist's advice would be effective.

Limitations - small sample size, low response rate for questionnaire

1 Royal Collage of Physicians, National clinical guidelines for stroke, October 2016

2 BIOS, BIOS position statement for visual services in stroke practice, 2014

${ }^{3}$ Akin Nihat, Jayesh Khistria, Lucy Reynolds, Arani Nitkunan, Improving detection and management of visual symptoms after stroke - an audit, 2014

${ }^{4}$ Rowe, Brand, Jackson et al. Visual impairment following stroke: do stroke patients require vision assessment? Age and Ageing 2009 